(C) 1982. The Genetical Society of Great Britain

\title{
ASSORTATIVE MATING WITH DOMINANCE
}

\author{
A. E. STARK \\ School of Community Medicine, The University of New South Wales, \\ P.O. Box 1, Kensington, Australia, 2033
}

Received 21.v.81

HALDANE (1924) introduced a model of assortative mating with dominance but gave only sufficient of its properties to demonstrate the influence of assortative mating on selection. Here it is compared with the much better understood model of O'Donald (1960).

Let the frequencies at generation $t$ of genotypes $g g, g G$, and $G G$ be denoted by $f_{0}(t), f_{1}(t)$, and $f_{2}(t)$, respectively. The first is referred to as the rec type and the others in combination as the dom type.

The mating frequencies in Haldane's system are

$$
\begin{aligned}
\text { rec female } \times \text { rec male: } & f_{0}(t)-\theta(t) \\
\text { rec female } \times \text { dom male: } & \theta(t) \\
\text { dom female } \times \text { rec male: } & \theta(t) \\
\text { dom female } \times \text { dom male: } & 1-f_{0}(t)-\theta(t),
\end{aligned}
$$

where $\theta(t)$ is constrained by the constant assortative mating parameter $\Lambda$ $(-1 \leqq \Lambda<\infty)$ according to

$$
\left(f_{0}(t)-\theta(t)\right)\left(1-f_{0}(t)-\theta(t)\right)=(1+\Lambda)(\theta(t))^{2} .
$$

Rearranging (2) gives

$$
\theta(t)=\left(\left\{1+4 \Lambda f_{0}(t)\left[1-f_{0}(t)\right]\right\}^{1 / 2}-1\right) /(2 \Lambda) .
$$

For purposes of analysis the mating frequencies are put in genotypic pairings whose frequencies are denoted by $f_{i j}(t)(i=0,1,2 ; j=0,1,2)$ which are expressed in canonical form

$$
f_{i j}(t)=f_{i}(t) \cdot f_{i}(t)\left(1+\rho(t) \cdot x_{i}(t), x_{i}(t)\right) .
$$

Then Haldane's frequencies (1) conform to (3) when:

$$
\begin{aligned}
\rho(t) & \equiv \rho^{*}(t)=1-\theta(t) /\left\{f_{0}(t)\left(1-f_{0}(t)\right)\right\} ; \\
x_{1}(t) & =x_{2}(t)=\left\{f_{0}(t) /\left(1-f_{0}(t)\right)\right\}^{1 / 2}, \quad x_{0}(t)=-\left(x_{1}(t)\right)^{-1} .
\end{aligned}
$$

In O'Donald's system a fixed proportion $\alpha$ of phenotypes mate with like phenotype and the remainder mate at random. The mating frequencies under this scheme can be put in form (3) by the substitution $\rho(t)=\rho \equiv \rho^{\prime}=\alpha$ and $\left\{x_{i}(t)\right\}$ as in (5).

In equilibrium the frequency of genotype $g G$ is given by O'Donald as

$$
f_{1}=p^{2}(\alpha-1)+p\left\{(1-\alpha)\left(p^{2}(1-\alpha)+4 q\right)\right\}^{1 / 2},
$$

where $q=f_{0}+\frac{1}{2} f_{1}$ and $p=1-q$. Using the corresponding $f_{0}$, the same mating, and therefore the same offspring frequencies, can be obtained in equilibrium under Haldane's scheme by setting $\Lambda$ to be

$$
\Lambda=\alpha /\left(f_{0}\left(1-f_{0}\right)(1-\alpha)^{2}\right),
$$


for then

$$
\theta(t)=\theta=f_{0}\left(1-f_{0}\right)(1-\alpha)
$$

and $\rho^{*}(t)=\rho^{*}=\alpha=\rho^{\prime}$.

The rate of approach to equilibrium is of the same order in the two systems.

The parameter $\rho$ can be expressed in terms of $q$ and $p$ and the index of homogamy $\lambda$, when $f_{0}=q^{2}+\lambda p q$ etc. From the equilibrium identity $f_{11}=4 f_{02}, \rho$ is found to be

$$
\rho=\lambda(1+q(1-\lambda)) /(q+\lambda p) .
$$

Equation (6), taken in conjunction with (3) and (5), shows that systems in which assortative mating is based on dominance and which give the same genotypic distribution, have identical mating frequencies in equilibrium.

Acknowledgments.-Thanks are due to Professors S. D. Jayakar and C. Matessi, CNR, Pavia, Italy, who read an earlier version of the manuscript and offered constructive comments.

\section{REFERENCES}

HALDANE, J. B. S. 1924. A mathematical theory of natural and artificial selection. Part II. The influence of partial self-fertilisation, inbreeding, assortative mating, and selective fertilisation on the composition of Mendelian populations, and on natural selection. Proc. Camb. Phil. Soc., Biol. Sc., 1, 158-163.

O'DONALD, P. 1960. Assortive mating in a population in which two alleles are segregating. Heredity, 15, 389-396. 